\title{
Mathematical Modeling of a Brain-on-a-Chip: A Study of the Neuronal Nitric Oxide Role in Cerebral Microaneurysms
}

\author{
Corina S. Drapaca ${ }^{a^{*}}$ \\ ${ }^{a}$ Department of Engineering Science and Mechanics, Pennsylvania State University, University Park, PA 16802, USA
}

\begin{abstract}
Brain tissue is a complex material made of interconnected neural, glial, and vascular networks. While the physics and biochemistry of brain's cell types and their interactions within their networks have been studied extensively, only recently the interactions of and feedback among the networks have started to capture the attention of the research community. Thus, a good understanding of the coupled mechano-electrochemical processes that either provide or diminish brain's functions is still lacking. One way to increase the knowledge on how the brain yields its functions is by developing a robust controlled feedback engineering system that uses fundamental science concepts to guide and interpret experiments investigating brain's response to various stimuli, aging, trauma, diseases, treatment and recovery processes. Recently, a mathematical model for an implantable neuro-glial-vascular unit, named brain-on-a-chip, was proposed that can be optimized to perform some fundamental cellular processes that could facilitate monitoring and supporting brain's functions, and highlight basic brain mechanisms. In this paper we use coupled elastic, viscoelastic and mass elements to model a brain-ona-chip made of a neuron and its membrane, and astrocyte's endfeet connected to an arteriole's wall. We propose two constrained Lagrangian formulations that link the Hodgkin-Huxley model of the neuronal membrane, and the mechanics of the neuron, neuronal membrane, and the glia's endfeet. The effects of the nitric oxide produced by neurons and endothelial cells on the proposed brain-on-a-chip are investigated through numerical simulations. Our numerical simulations suggest that a non-decaying synthesis of nitric oxide may contribute to the onset of a cerebral microaneurysm.
\end{abstract}

\section{Keywords:}

Mechano-Electrochemical Model;

Hamilton's Principle;

Hodgkin-Huxley Model;

Nitric Oxide Dynamics;

Neuro-Glial-Vascular Unit.

\section{Article History:}

Received: 18 October 2018

Accepted: 02 December 2018

\section{1- Introduction}

Brain tissue is an inhomogeneous multi-phase material composed of interconnected neural, glial, and vascular networks. So far, the physics and biochemistry of brain's cell types and their interactions within their respective networks have been extensively studied. However, a full understanding of brain's physics and biochemistry comes not only from an integrated analysis of the cells within their networks, but also from the interactions of and feedback among the networks. Although in recent years studies on biochemical interactions among brain's networks have started to emerge [1-7], a good comprehension of the coupled mechano-electrochemical processes that either provide or diminish brain's functions is still lacking. This knowledge is critical in improving existing diagnostic and therapeutic protocols and developing better new ones.

One way to deepen the understanding of how the brain yields its functions is by developing a robust controlled feedback engineering system that uses fundamental science concepts to guide and interpret experiments investigating brain's response to various stimuli, aging, trauma, diseases, treatment and recovery processes. An example of such an engineering system is the brain-on-a-chip proposed in [8]. A brain-on-a-chip is envisioned as an implantable, humanmade neuro-glial-vascular unit that couples certain fundamental mechano-electrochemical mechanisms of its constituents such that it could provide functional support after a stroke or serious traumatic injury. Specialized sensors could be added on the chip to allow it to recognize biomarkers of brain diseases and transmit information about an imminent traumatic event to a receiver outside the head. In addition, a brain-on-a-chip may be adapted for targeted drug delivery to a brain's region aff ected by disease. Lastly, the chip could also be used to highlight possible brain's

\footnotetext{
*CONTACT: Csd12@psu.edu

DOI: http://dx.doi.org/10.28991/esj-2018-01156
}

(C) This is an open access article under the CC-BY license (https://creativecommons.org/licenses/by/4.0/). 
mechanisms of health and disease which are otherwise very difficult to observe in a living brain. It is important to notice that this concept of an engineered brain-on-a-chip is different from the very few recently developed brain-on-a-chip technologies that aim to reproduce only neural networks in vitro for pharmaceutical purposes [9-10].

At this stage we are interested in the mathematical modelling of a brain-on-a-chip optimized to study the possible role played by the neuronal nitric oxide in the onset of cerebral microaneurysms. Figure 1 shows a schematic of those aspects of the neuro-glial-vascular unit that are of interest to this paper. A neuro-glial-vascular unit (also known in the literature as neurovascular unit) is made of a group of neurons and nearby astrocytes (glial cells) that are connected functionally to the endothelium and smooth muscles of the small blood vessels with the purpose of controlling the supply of cerebral blood needed for the proper functionality of the brain [2]. Astrocytes have at least one endfoot that is in contact with the vasculature, and it is believed that the endfeet of all astrocytes completely encompass all the cerebral vessels [4]. Besides absorbing much needed nutrients (especially glucose) from the blood, the endfeet provide bidirectional control of arterial diameter by both constricting and dilating arterioles [3-4]. Gordon et al [3] and Petzold and Murthy [6], among others, suggested that there are two separate endfeet in contact with an arteriole, one that is responsible for vasodilatation and the other for vasoconstriction. This suggests that the blood flow in the small cerebral vessels is not only a pulsatile motion due to the mechanics of the heart but also driven by biochemical processes specific to the brain. Nitric oxide and brain metabolic elements such as oxygen, lactate and adenosine, are known to play a role in the dilatation and constriction of vessels by astrocytes' endfeet [4]. In particular, numerous studies have shown that nitric oxide (NO), a small diffusible molecule, is essential to neuro-glial-vascular signalling and regulation of cerebral blood flow [1-2, 5, 11-18]. In the brain, $\mathrm{NO}$ is produced by synthesis of three isoforms. Two isoforms are $\mathrm{Ca}^{2+}$ dependent and are expressed in the neurons (nNO) and in the vascular endothelium and choroid plexus (eNO) [14]. The third isoform is inducible and independent of calcium (iNO) and is expressed in macrophages, glial cells, and tumour cells in response to insults such as ischemia and neuroinflammation [2, 14]. Endothelial NO maintains cerebral microcirculation by inhibiting platelet aggregation, leukocyte adhesion and migration, and reducing the blood pressure and the proliferation of the vascular smooth muscle. This isoform (eNO) is the only one known to be activated, among others, by the shear stress at the interface between the vessel wall and the blood flow [14]. Neuronal NO is a neurotransmitter essential to neuronal plasticity, memory formation, and regulation of the cerebral blood flow [14]. Details about the biochemical processes by which NO controls vasomotor responses can be found for instance in [1, 3$4,14]$. Briefly, NO is considered a vasodilating agent which can also inhibit the production of other vasoconstricting and vasodilating agents. Lastly, for the sake of completeness, we mention that NO is also formed in the red blood cells and blood's hemoglobin scavenges some of the produced NO [16].

In this paper we propose two mathematical models of brain-on-a-chip corresponding to the neuro-glial-vascular unit shown in Figure 1. The models are used to study through numerical simulations the effects of $\mathrm{Ca}^{2+}$ - dependent NO on the chip. More precisely, we use the two models to study the possible role played by NO in the onset of a microaneurysm. Thus, in this paper the suggested theoretical concept of a brain-on-a-chip is utilized to learn about one viable mechanism of cerebral microaneurysms. In both models the neuron and its membrane are represented as in [19-20]: the neuron is a linear viscoelastic Kelvin-Voigt solid, and linear viscoelastic Maxwell fluid elements are used to model the ionic gates $m, n$, and $h$ of the neuronal membrane introduced by the Hodgkin-Huxley model. The astrocyte is represented by either one endfoot controlling vasodilatation (model 1) or two endfeet with the endfeet regulating opposite vasomotor responses (model 2). The endfoot responsible for vasodilatation is modeled as a spring-mass system and is coupled to the viscoelastic and mass elements representing the neuron and its membrane. Two forces of opposite directions that are proportional to the concentration of NO act on the neuron and the endfoot regulating vasodilatation. These rheological elements are linked to the Hodgkin-Huxley electrical circuit of the neuronal membrane using constrained Lagrangian formulations. The corresponding Euler-Lagrange equations for the two models are obtained from the non-conservative form of Hamilton's principle. The effects of NO produced by neurons and endothelial cells on the mechanoelectrochemical behavior of the two brain-on-a-chip are investigated through numerical simulations. Our results suggest that a non-decaying synthesis of NO may be a contributing factor in the formation and growth of a microaneurysm. This finding indicates that a microaneurysm's onset may be caused by a combination of deviant mechanical pushing of the vascular wall by the blood flow and anomalous chemically-induced pulling of the wall exerted by the neuro-glialvascular unit. A more in-depth study of this hypothesis will be the focus of future work.

The paper is organized as follows. In the next section we provide two designs and corresponding mathematical models for a brain-on-a-chip. A brief review of two mathematical models proposed by Hall and Garthwaite [15] that describe the NO dynamics in brain with non-decaying and, respectively, dynamic NO synthesis, is also given in section 2. Section 3 presents our results, followed by a discussion of the results in section 4 . The paper ends with a section containing conclusions and future work. 


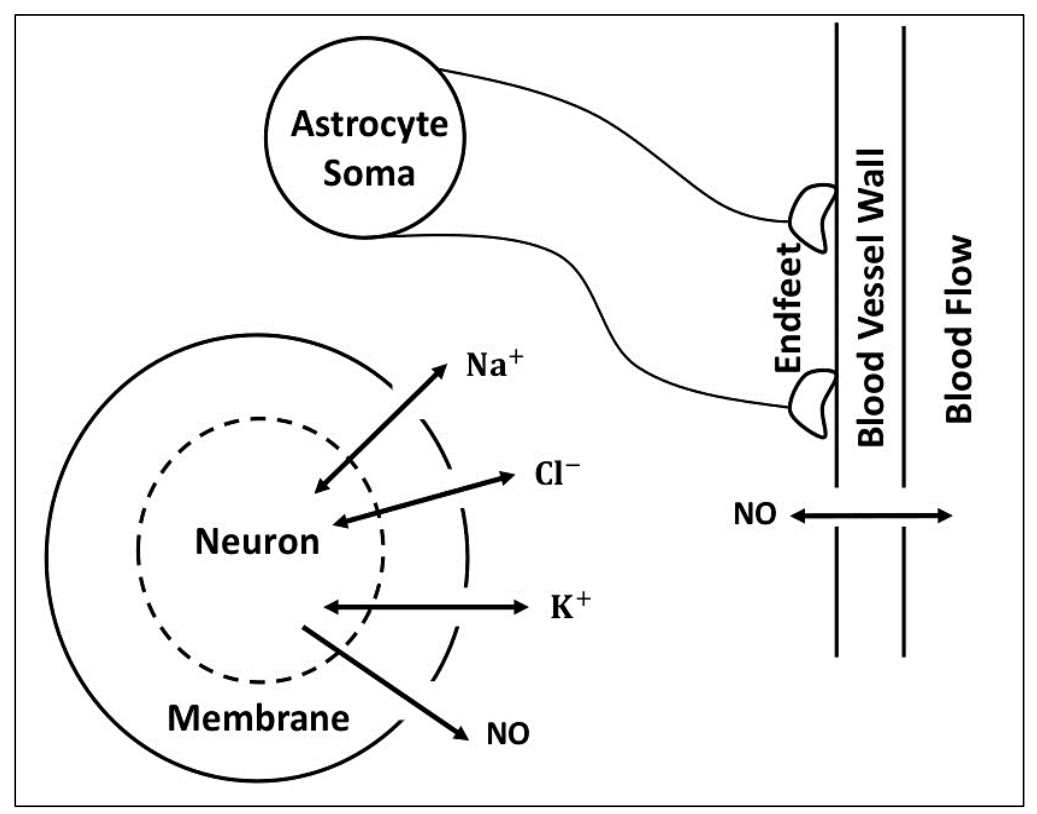

Figure 1. A drawing of the neuro-glial-vascular unit showing only the structure and components of interest to this work. Astrocyte's endfeet use NO (among other chemical species) to control opposite vasomotor responses. The diffusive NO is produced by neurons, endothelial and red blood cells. An action potential (the fast moving of $\mathrm{Na}^{+}, \mathrm{K}^{+}$, and $\mathrm{Cl}^{-}$across neuronal membrane) due to an externally applied current affects the production of neuronal NO and causes a mechanical deformation of the neuron. Because of the very close proximity of the neuron and astrocyte, the mechanical deformation of the neuron triggers a mechanical movement of astrocyte's endfeet. (Drawing inspired by diagrams published in [1, 4-5] that depict various biological pathways facilitating the functions of the neuro-glial-vascular unit.)

\section{2- Mathematical Models}

As in [8, 19-20], the neuron is modelled as an axi-symmetric circular cylindrical annulus. Neuron's intracellular space fills the inner core of radius $r_{0}$, while the outer core of thickness $r \ll r_{0}$ represents neuronal membrane (Figure 2 ). The deformation of the neuron is only along the radial direction and we denote the independent spatial variable by $X$. The center of the neuron, $X=0$, is assumed to be fixed, and the interface between the intracellular space and the membrane, $X=r_{0}$, is moving due to ionic transport across the membrane, NO dynamics, and mechanical interactions between the neuron and astrocyte. We further assume that the neuron's intracellular space and membrane are homogeneous materials and thus we can use the lower-dimensional electromechanical element introduced in [19-20] and drawn in Figure 2. This electromechanical element couples the following: 1) a linear viscoelastic Kelvin-Voigt solid element representing the intracellular space, 2) linear viscoelastic Maxwell fluid elements located in the membrane that model the ionic gates $m, n$, and $h$ introduced by the Hodgkin-Huxley model [21], and 3) the electric circuit model of the membrane proposed by Hodgkin and Huxley [21]. Although the Hodgkin-Huxley electric circuit and the set of Maxwell elements enriched with a complex chemo-mechanical behavior are equivalent, for simplicity, in this paper we consider them to be independent. The astrocyte is represented only by its endfeet that control the vasodilatation and vasoconstriction of a blood vessel wall. The endfoot responsible for vasodilatation is modelled as a spring-mass system connected in series to the Kelvin-Voigt viscoelastic element - mass system representing the neuron. The compression of the spring in this spring-mass system corresponds to vasodilatation. For now, we neglect the chemo-mechanical processes involved in vasoconstriction and assume that vasoconstriction is a purely mechanical consequence of vasodilatation. Thus, the vasoconstrictive endfoot is modelled as a mass connected to the astrocyte's soma by a massless rigid rope. Our model of an astrocyte with two endfeet is an analog of an Atwood machine where the astrocyte's soma acts like a massless frictionless pulley and the weight forces are replaced by other forces relevant to the problem considered here. The two masses of the endfeet are assumed to be attached to the vascular wall so that their dynamics could be identified with the motion of the vessel itself. The neuron and astrocyte's endfeet are exposed to forces proportional to the concentration of NO. For now, we neglect the dynamics of other ions and species that facilitate the interactions among the neuron, astrocyte, and vascular wall. In addition, we also neglect the interactions of brain cells with the extracellular fluid since the width of the extracellular space in the brain (order of magnitude of $\mathrm{nm}$ ) is much smaller than the radii of the cells (order of magnitude of $\mu \mathrm{m}$ ) [22]. Because we are interested here in the linkage between neuronal electromechanics and vasodilatation via glia's endfeet, we investigate the mechanical behavior of the coupled neuro-glia in two cases: 1) the astrocyte has only one endfoot controlling vasodilatation (subsection 2.1), and 2) the astrocyte has two endfeet (subsection 2.2). The coupling of the electric and mechanical elements mentioned above is achieved by using Lagrangian formulations and Hamilton's principle. 


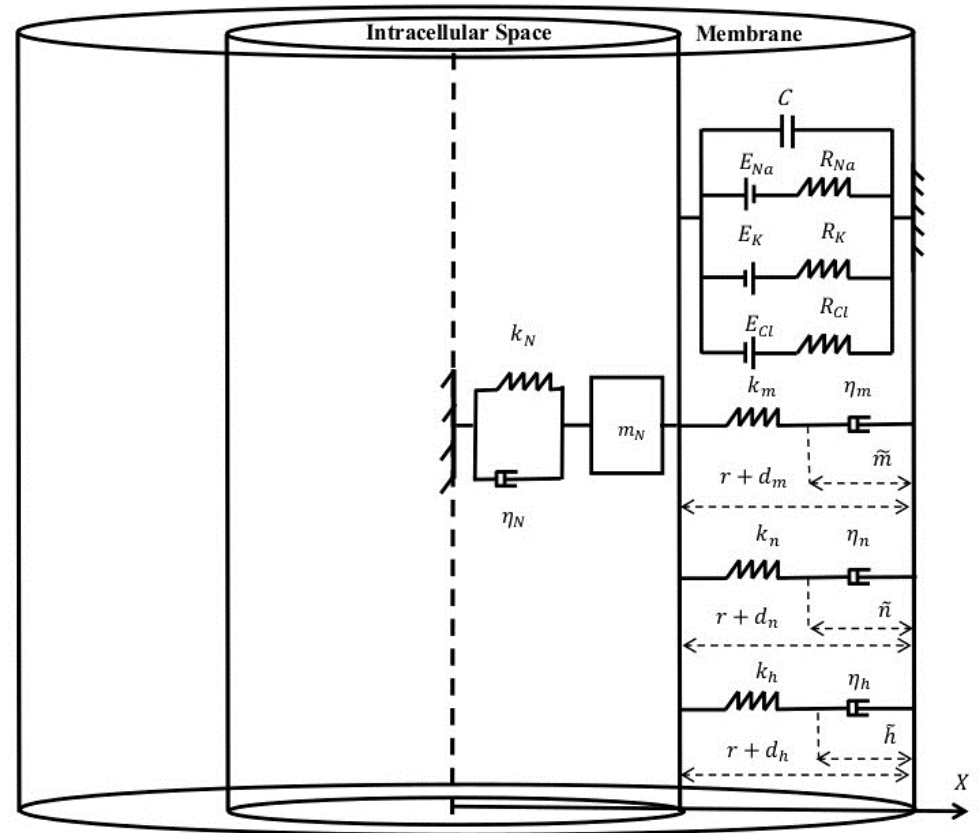

Figure 2. Schematic of the electromechanical model of a neuron and its membrane: the neuron is a homogeneous axisymmetric circular cylinder whose inner core is filled with the intracellular space and the outer layer is the membrane. Because of the geometrical symmetry and material homogeneity, it is enough to study half of the neuron modelled as a spring-dashpot-mass mechanical system with the spring and dashpot connected in parallel (linear viscoelastic Kelvin-Voight element). The membrane is represented as an electrical circuit described by the Hodgkin-Huxley equations where the ionic gates $\boldsymbol{m}, \boldsymbol{n}$, and $\boldsymbol{h}$ are modelled as linear viscoelastic Maxwell elements connected in parallel.

In what follows, we denote the first order derivative with respect to the time variable $t$ of a generic differentiable function $f(t)$ by either $\dot{f}$ or $d f / d t$.

\section{2-1-Astrocyte with One Endfoot}

A schematic of this case is shown in Figure 3.

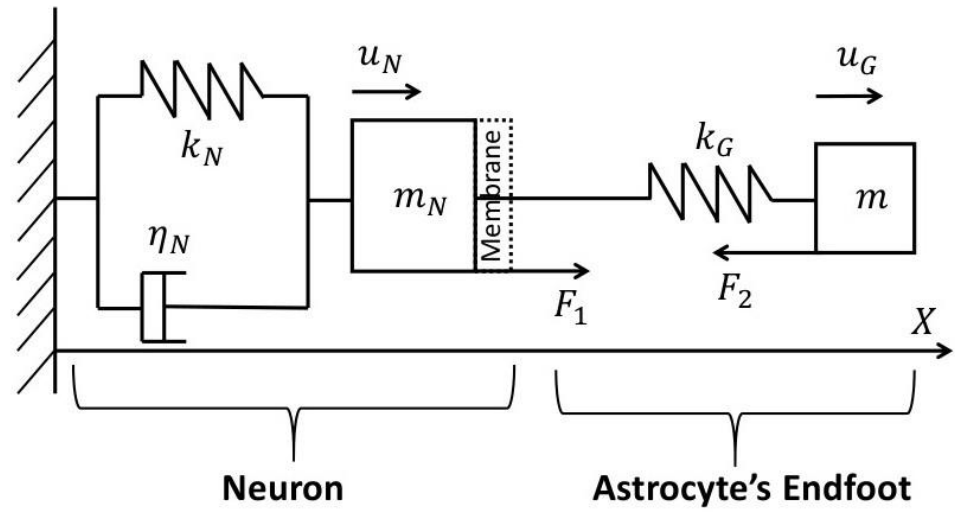

Figure 3. Schematic of a brain-on-a-chip made of a coupled neuron (modeled as a linear viscoelastic Kelvin-Voigt element connected to a mass) and astrocyte's endfoot (modeled as a spring-mass mechanical system). Forces $F_{1}$ and $F_{2}$ are assumed to be proportional to the concentration of NO. The opposite directions of these forces compress the spring in the spring-mass system which is assumed to model vasodilatation. The deformation of the neuron is caused by the action of the applied force $F_{1}$ and the mechanical response of the neuron to action potentials triggered by an externally applied current $I$.

The Lagrangian form of this model is:

$$
\begin{aligned}
& \mathcal{L}_{1}\left(u_{N}, u_{G}, q_{C}, \tilde{m}, \tilde{n}, \tilde{h}\right)=\frac{1}{2} m_{N} \dot{u}_{N}{ }^{2}+\frac{1}{2} m_{G} \dot{u}_{G}{ }^{2}-\frac{1}{2} k_{N}(\tilde{m}, \tilde{n}, \tilde{h}) u_{N}{ }^{2}-\frac{1}{2} k_{G}\left(u_{G}-u_{N}\right)^{2} \\
& -\frac{1}{2 C\left(u_{N}\right)} q_{C}{ }^{2}-\frac{1}{2} k_{m}\left(r+d_{m}-\tilde{m}\right)^{2}-\frac{1}{2} k_{n}\left(r+d_{n}-\tilde{n}\right)^{2}-\frac{1}{2} k_{h}\left(r+d_{h}-\tilde{h}\right)^{2},
\end{aligned}
$$


where $m_{N}$ is half of the constant mass of the neuron with constant cross-sectional area $A, u_{N}(t)$ is the macroscopic (cell scale) displacement of $m_{N}, m_{G}$ is the constant mass of the astrocyte's endfoot, $u_{G}(t)$ is the macroscopic (cell scale) displacement of $m_{G}, r+d_{m}, r+d_{n}$, and $r+d_{h}$ are the relative displacements of the Maxwell elements, $\tilde{m}, \tilde{n}$, and $\tilde{h}$ are the microscopic (ionic scale) displacements of the dashpots in the Maxwell elements. The spring constant of the Kelvin-Voigt element is denoted by $k_{N}$, while $k_{m}, k_{n}$, and $k_{h}$ are the spring constants of the corresponding Maxwell elements. Lastly, $C$ is the macroscopic capacitance of membrane's lipid bilayer modeled as a capacitor of electric charge $q_{C}$. For simplicity, we assume that the relative displacements $d_{m}, d_{n}$, and $d_{h}$ are independent of displacements $u_{N}$ and $u_{G}$. The Lagrangian form $\mathcal{L}_{1}$ given by formula (1) is a combination of macro-kinetic mechanical energies (the first and second terms), macro-potential mechanical energies (the third and fourth terms), a macro-potential electric energy (the fifth term), and micro-potential mechanical energies (the last three terms).

Following the variational formulations in [8, 19-20], we introduce $q_{N a}, q_{K}$, and $q_{C l}$ the electric charges of $N a^{+}, K^{+}$, and $\mathrm{Cl}^{-}$channels that are considered in the Hodgkin-Huxley model. The conservation of electric charges gives the following constraint:

$q_{C}+q_{N a}+q_{K}+q_{C l}=0$.

Let $u_{N}, u_{G}, q_{N a}, q_{K}, q_{C l}, \tilde{m}, \tilde{n}$, and $\tilde{h}$ be generalized coordinates. If we replace $q_{C}$ from formula (2) into the expression (1) of the Lagrangian $\mathcal{L}_{1}$, then we can calculate the variation of the Lagrangian $\delta \mathcal{L}_{1}$ as follows:

$$
\begin{aligned}
& \delta \mathcal{L}_{1}=\lim _{\varepsilon \rightarrow 0} \frac{d \mathcal{L}_{1}}{d \varepsilon}\left(u_{N}+\varepsilon \delta u_{N}, u_{G}+\varepsilon \delta u_{G}, q_{N a}+\varepsilon \delta q_{N a}, q_{K}+\varepsilon \delta q_{K}, q_{C l}+\varepsilon \delta q_{C l}, \tilde{m}+\varepsilon \delta \tilde{m},\right. \\
& \tilde{n}+\varepsilon \delta \tilde{n}, \tilde{h}+\varepsilon \delta \tilde{h})=m_{N} \dot{u}_{N} \delta \dot{u}_{N}+m_{G} \dot{u}_{G} \delta \dot{u}_{G}+\left(-k_{N} u_{N}+k_{G}\left(u_{G}-u_{N}\right)+\frac{1}{2} V^{2} \frac{d C}{d u_{N}}\right) \delta u_{N} \\
& -k_{G}\left(u_{G}-u_{N}\right) \delta u_{G}+V\left(\delta q_{N a}+\delta q_{K}+\delta q_{C l}\right)+\left(-\frac{1}{2} \frac{\partial k_{N}}{\partial \tilde{m}} u_{N}^{2}+k_{m}\left(r+d_{m}-\tilde{m}\right)\right) \delta \tilde{m} \\
& +\left(-\frac{1}{2} \frac{\partial k_{N}}{\partial \tilde{n}} u_{N}^{2}+k_{n}\left(r+d_{n}-\tilde{n}\right)\right) \delta \tilde{n}+\left(-\frac{1}{2} \frac{\partial k_{N}}{\partial \tilde{h}} u_{N}^{2}+k_{h}\left(r+d_{h}-\tilde{h}\right)\right) \delta \tilde{h},
\end{aligned}
$$

where $\delta u_{N}, \delta u_{G}, \delta q_{N a}, \delta q_{K}, \delta q_{C l}, \delta \tilde{m}, \delta \tilde{n}$, and $\delta \tilde{h}$ are linearly independent variations of the corresponding generalized coordinates. In formula (3), $V=q_{C} /_{C}$ is the electric potential of the capacitor.

The virtual work done by non-conservative forces is:

$$
\begin{aligned}
& \delta W_{1}=-\left(R_{N a} \dot{q}_{N a} \delta q_{N a}+R_{K} \dot{q}_{K} \delta q_{K}+R_{C l} \dot{q}_{C l} \delta q_{C l}\right)-\left(E_{N a} \delta q_{N a}+E_{K} \delta q_{K}+E_{C l} \delta q_{C l}\right) \\
& -\left(\eta_{N} \dot{u}_{N} \delta u_{N}+\eta_{m} \dot{\tilde{m}} \delta \tilde{m}+\eta_{n} \dot{\tilde{n}} \delta \tilde{n}+\eta_{h} \dot{\tilde{h}} \delta \tilde{h}\right)+F_{1} \delta u_{N}+F_{2} \delta u_{G} .
\end{aligned}
$$

The terms in the first set of parentheses in formula (4) are dissipative forces due to the resistors of resistances $R_{N a}$, $R_{K}$, and $R_{C l}$ in the Hodgkin-Huxley electric circuit. The second set of parentheses contains the reverse potentials $E_{N a}$, $E_{K}$, and $E_{C l}$ of the Hodgkin-Huxley model. The terms in the third set of parentheses are dissipative forces due to the linear dashpots of the Kelvin-Voigt and Maxwell elements with damping coefficients $\eta_{N}, \eta_{m}$, $\eta_{n}$, and $\eta_{h}$. Lastly, the forces $F_{1}$ and $F_{2}$ in formula (4) are work conjugates for the Kelvin-Voigt element and respectively the spring-mass system representing astrocyte's endfoot. The signs in formula (4) are chosen such that the virtual work $\delta W_{1}$ is thermodynamically consistent.

We apply now the non-conservative form of Hamilton's principle:

$\int_{t_{1}}^{t_{2}}\left(\delta \mathcal{L}_{1}+\delta W_{1}\right) d t=0$,

Where $t_{1}$ and $t_{2}$ are arbitrary times where the variations $\delta u_{N}, \delta u_{G}, \delta q_{N a}, \delta q_{K}, \delta q_{C l}, \delta \tilde{m}, \delta \tilde{n}$, and $\delta \tilde{h}$ vanish.

By replacing formulas (3) and (4) into (5), integrating by parts, and using the linear independence of the variations of the generalized coordinates and the fact that they are zero at $t_{1}$ and $t_{2}$, we obtain the following Euler-Lagrange equations:

$$
\begin{aligned}
& -m_{N} \ddot{u}_{N}-\eta_{N} \dot{u}_{N}-k_{N} u_{N}+k_{G}\left(u_{G}-u_{N}\right)+\frac{1}{2} V^{2} \frac{d C}{d u_{N}}+F_{1}=0 \\
& -m_{G} \ddot{u}_{G}-k_{G}\left(u_{G}-u_{N}\right)+F_{2}=0 \\
& -R_{N a} \dot{q}_{N a}-E_{N a}+V=0 \\
& -R_{K} \dot{q}_{K}-E_{K}+V=0 \\
& -R_{C l} \dot{q}_{C l}-E_{C l}+V=0
\end{aligned}
$$




$$
\begin{aligned}
& -\eta_{m} \dot{\tilde{m}}+k_{m}\left(r+d_{m}-\tilde{m}\right)-\frac{1}{2} \frac{\partial k_{N}}{\partial \widetilde{m}} u_{N}^{2}=0 \\
& -\eta_{n} \dot{\tilde{n}}+k_{n}\left(r+d_{n}-\tilde{n}\right)-\frac{1}{2} \frac{\partial k_{N}}{\partial \tilde{n}} u_{N}^{2}=0 \\
& -\eta_{h} \dot{\tilde{h}}+k_{h}\left(r+d_{h}-\tilde{h}\right)-\frac{1}{2} \frac{\partial k_{N}}{\partial \widetilde{h}} u_{N}^{2}=0
\end{aligned}
$$

Lastly, Kirchhoff's current law needs to be added to the system of equations (6) - (13):

$\frac{d}{d t}\left(C V+q_{N a}+q_{K}+q_{C l}\right)=I$,

Where $I$ is a known external current applied on the membrane. By replacing the expressions of $\dot{q}_{N a}, \dot{q}_{K}$, and $\dot{q}_{C l}$ from equations (8) - (10) in equation (14) we obtain:

$C \dot{V}+\frac{d C}{d u_{N}} \dot{u}_{N} V=I-\frac{1}{R_{N a}}\left(V-E_{N a}\right)-\frac{1}{R_{K}}\left(V-E_{K}\right)-\frac{1}{R_{C l}}\left(V-E_{C l}\right)$.

We introduce the non-dimensional displacements:

$m=\frac{\tilde{m}}{r}, n=\frac{\tilde{n}}{r}, h=\frac{\tilde{h}}{r}$

And re-write equations (11)-(13) as:

$\dot{m}=-\frac{k_{m}}{\eta_{m}} m+\left[\frac{k_{m}}{\eta_{m}}\left(1+\frac{d_{m}}{r}\right)-\frac{1}{2 r^{2} \eta_{m}} \frac{\partial k_{N}}{\partial m} u_{N}{ }^{2}\right]$

$\dot{n}=-\frac{k_{n}}{\eta_{n}} n+\left[\frac{k_{n}}{\eta_{n}}\left(1+\frac{d_{n}}{r}\right)-\frac{1}{2 r^{2} \eta_{n}} \frac{\partial k_{N}}{\partial n} u_{N}^{2}\right]$

$\dot{h}=-\frac{k_{h}}{\eta_{h}} h+\left[\frac{k_{h}}{\eta_{h}}\left(1+\frac{d_{h}}{r}\right)-\frac{1}{2 r^{2} \eta_{h}} \frac{\partial k_{N}}{\partial h} u_{N}^{2}\right]$

By noticing that equations (16) - (18) have the same forms as the classic Hodgkin-Huxley equations for the gating variables $m, n$, and $h$, we will further identify our non-dimensional displacements $m, n$, and $h$ with the variables representing the activations of the $\mathrm{Na}^{+}$and $\mathrm{K}^{+}$channels and the inactivation of $\mathrm{Na}^{+}$channel, respectively.

The physical parameters required by equations (16) - (18) are not known yet and therefore we will replace the righthand sides of these equations by the expressions from the modified Hodgkin-Huxley model proposed in [7]. The very finely adjusted equations and parameters of the Hodgkin-Huxley model combined with the lack of knowledge of the function $C\left(u_{N}\right)$ makes the system of differential equations very difficult to solve using existing numerical solvers. For now, we resolved this issue by neglecting the terms involving $\frac{d C}{d u_{N}}$ in equations (6) and (15). Thus, the system of equations corresponding to this model is:

$$
\begin{aligned}
& m_{N} \ddot{u}_{N}+\eta_{N} \dot{u}_{N}+k_{N} u_{N}-k_{G}\left(u_{G}-u_{N}\right)-F_{1}=0 \\
& m_{G} \ddot{u}_{G}+k_{G}\left(u_{G}-u_{N}\right)-F_{2}=0 \\
& \dot{m}=\alpha_{m}(1-m)-\beta_{m} m \\
& \dot{n}=\alpha_{n}(1-n)-\beta_{n} n \\
& \dot{h}=\alpha_{h}(1-h)-\beta_{h} h \\
& C \dot{V}=I-\left(G_{N a} m^{3} h+G_{N a L}\right) \tilde{A}\left(V-E_{N a}\right) \\
& -\left(G_{K} n^{4}+G_{K L}\right) \tilde{A}\left(V-E_{K}\right)-G_{C l L} \tilde{A}\left(V-E_{C l}\right)
\end{aligned}
$$

Where $\tilde{A}$ is the surface area of the neuron, and the constant parameters $G_{N a}, G_{K}, G_{N a L}, G_{K L}$, and $G_{C l L}$ represent respectively the $\mathrm{Na}^{+}$voltage-gated maximal conductance, the $\mathrm{K}^{+}$voltage-gated maximal conductance, the $\mathrm{Na}^{+}$leak conductance, the $\mathrm{K}^{+}$leak conductance, and the $\mathrm{Cl}^{-}$leak conductance. Also:

$$
\begin{array}{ll}
\alpha_{m}=\frac{0.32(V+54)}{1-\exp (-0.25(V+54))}, & \beta_{m}=\frac{0.28(V+27)}{\exp (0.2(V+27))-1} \\
\alpha_{n}=\frac{0.032(V+52)}{1-\exp (-0.2(V+52))}, & \beta_{n}=0.5 \exp \left(-\frac{V+57}{40}\right)
\end{array}
$$




$$
\alpha_{h}=0.128 \exp \left(-\frac{V+50}{18}\right), \beta_{h}=\frac{4}{1+\exp (-0.2(V+27))}
$$

In system (19) - (24), the coupling between the Hodgkin-Huxley model and the mechanical behaviour of the neuron is achieved through the expressions of $k_{N}(m, n, h)$ and $C\left(u_{N}\right)$. As in [19-20], the neuronal membrane is assumed to act like a parallel plate capacitor and define:

$C=c_{m} \tilde{A}=\frac{\epsilon \tilde{A}}{r\left(1+\frac{u_{N}}{r}\right)} \approx \frac{\epsilon \tilde{A}}{r}\left(1-\frac{u_{N}}{r}\right)$,

Where $c_{m}$ and $\epsilon$ are the specific capacitance and, respectively, permittivity of the membrane. The dynamic spring constant is taken to be [9-10]:

$k_{N}(m, n, h)=k_{0}\left(1+m^{3}(1-h) n^{4}\right)$,

With $k_{0}$ the spring constant of the neuron in the state of rest. Formula (27) suggests that the neuron stiffens during an action potential which agrees with the experimental observations in [23-24].

\section{2-2-Astrocyte with Two Endfeet}

A schematic of this case is shown in Figure 4. The Lagrangian form of this model is:

$$
\begin{aligned}
& \mathcal{L}_{2}\left(u_{N}, u_{G}, x, q_{C}, \tilde{m}, \tilde{n}, \tilde{h}\right)=\frac{1}{2} m_{N} \dot{u}_{N}^{2}+\frac{1}{2} m_{G} \dot{x}^{2}+\frac{1}{2} m_{G}\left(\dot{u}_{G}-\dot{x}\right)^{2}-\frac{1}{2} k_{N}(\tilde{m}, \tilde{n}, \tilde{h}) u_{N}{ }^{2} \\
& -\frac{1}{2} k_{G}\left(d-x+u_{G}-u_{N}\right)^{2}-\frac{1}{2 C\left(u_{N}\right)} q_{C}{ }^{2} \\
& -\frac{1}{2} k_{m}\left(r+d_{m}-\tilde{m}\right)^{2}-\frac{1}{2} k_{n}\left(r+d_{n}-\tilde{n}\right)^{2}-\frac{1}{2} k_{h}\left(r+d_{h}-\tilde{h}\right)^{2},
\end{aligned}
$$

Where $x$ is the distance of the vasoconstrictive endfoot from the top of astrocyte's soma, and $d$ is the constant sum of the lengths of the endfeet at mechanical equilibrium. Thus $d-x$ is the distance from the top of the soma to the spring of constant $k_{G}$ at equilibrium. All the other parameters and variables in formula (28) are the same as those used in formula (1).

The generalized coordinates are now $u_{N}, u_{G}, x, q_{N a}, q_{K}, q_{C l}, \tilde{m}, \tilde{n}$, and $\tilde{h}$, with the corresponding linearly independent variations $\delta u_{N}, \delta u_{G}, \delta x, \delta q_{N a}, \delta q_{K}, \delta q_{C l}, \delta \tilde{m}, \delta \tilde{n}$, and $\delta \tilde{h}$. Using again formula (2) and the definition of the electric potential of the capacitor $V$, we can calculate the variation of the Lagrangian given by formula (28):

$$
\begin{aligned}
& \delta \mathcal{L}_{2}=m_{N} \dot{u}_{N} \delta \dot{u}_{N}+m_{G}\left(\dot{u}_{G}-\dot{x}\right) \delta \dot{u}_{G}+m_{G}\left(2 \dot{x}-\dot{u}_{G}\right) \delta \dot{x} \\
& +\left(-k_{N} u_{N}+k_{G}\left(d-x+u_{G}-u_{N}\right)+\frac{1}{2} V^{2} \frac{d C}{d u_{N}}\right) \delta u_{N}-k_{G}\left(d-x+u_{G}-u_{N}\right)\left(\delta u_{G}-\delta x\right) \\
& +V\left(\delta q_{N a}+\delta q_{K}+\delta q_{C l}\right)+\left(-\frac{1}{2} \frac{\partial k_{N}}{\partial \tilde{m}} u_{N}^{2}+k_{m}\left(r+d_{m}-\tilde{m}\right)\right) \delta \tilde{m} \\
& \quad+\left(-\frac{1}{2} \frac{\partial k_{N}}{\partial \tilde{n}} u_{N}{ }^{2}+k_{n}\left(r+d_{n}-\tilde{n}\right)\right) \delta \tilde{n}+\left(-\frac{1}{2} \frac{\partial k_{N}}{\partial \tilde{h}} u_{N}{ }^{2}+k_{h}\left(r+d_{h}-\tilde{h}\right)\right) \delta \tilde{h} .
\end{aligned}
$$

The virtual work in this case is:

$$
\begin{aligned}
& \delta W_{2}=-\left(R_{N a} \dot{q}_{N a} \delta q_{N a}+R_{K} \dot{q}_{K} \delta q_{K}+R_{C l} \dot{q}_{C l} \delta q_{C l}\right)-\left(E_{N a} \delta q_{N a}+E_{K} \delta q_{K}+E_{C l} \delta q_{C l}\right) \\
& -\left(\eta_{N} \dot{u}_{N} \delta u_{N}+\eta_{m} \dot{\tilde{m}} \delta \tilde{m}+\eta_{n} \dot{\tilde{n}} \delta \tilde{n}+\eta_{h} \dot{\tilde{h}} \delta \tilde{h}\right)+F_{1} \delta u_{N}+F_{2} \delta u_{G}+F_{3} \delta x .
\end{aligned}
$$

The meanings of the terms in formula (30) are the same as those in formula (4). The only difference between formulas (30) and (4) is an extra force $F_{3}$ in the expression of $\delta W_{2}$ which is a work conjugate for the second endfoot (see Figure $4)$. 


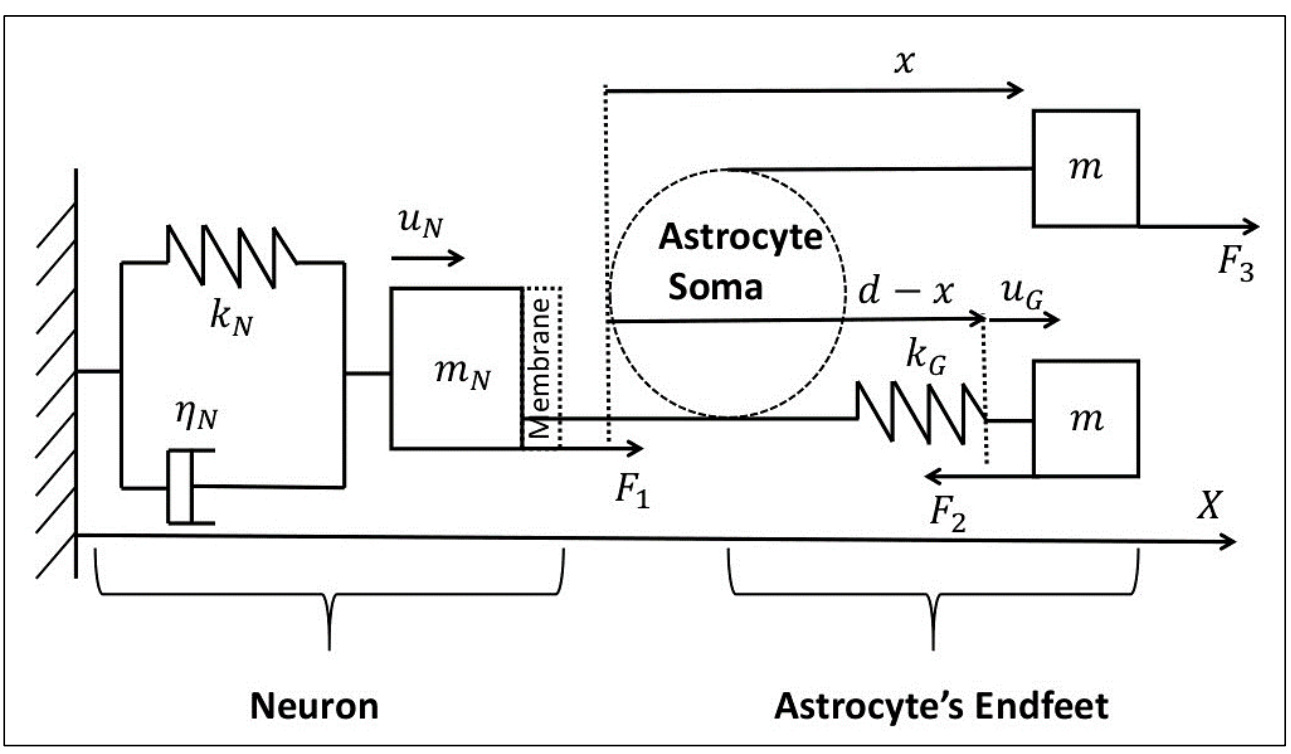

Figure 4. Schematic of a brain-on-a-chip made of a coupled neuron (modeled as a linear viscoelastic Kelvin-Voigt element connected to a mass) and astrocyte's endfeet. The endfeet are modeled as a spring-mass mechanical system and another mass connected to the astrocyte's soma by a frictionless massless wire. In this mechanical system, the astrocyte's soma acts as a massless frictionless pulley.

By applying again Hamilton's principle (5) for $\delta \mathcal{L}_{2}$ and $\delta W_{2}$ we obtain the following Euler-Lagrange equations corresponding to the variations $\delta u_{N}, \delta u_{G}$, and $\delta x$ :

$$
\begin{aligned}
& -m_{N} \ddot{u}_{N}-\eta_{N} \dot{u}_{N}-\left(k_{N}+k_{G}\right) u_{N}+k_{G}\left(d-x+u_{G}\right)+\frac{1}{2} V^{2} \frac{d C}{d u_{N}}+F_{1}=0 \\
& -2 m_{G} \ddot{x}+m_{G} \ddot{u}_{G}+k_{G}\left(d-x+u_{G}-u_{N}\right)+F_{3}=0 \\
& -m_{G} \ddot{u}_{G}+m_{G} \ddot{x}-k_{G}\left(d-x+u_{G}-u_{N}\right)+F_{2}=0
\end{aligned}
$$

The Euler-Lagrange equations corresponding to the variations $\delta q_{N a}, \delta q_{K}, \delta q_{C l}, \delta \tilde{m}, \delta \tilde{n}$, and $\delta \tilde{h}$ are again equations (8) - (13).

By adding equations (32) and (33) we obtain an equation only for the unknown $x(t)$ :

$m_{G} \ddot{x}=F_{2}+F_{3}$

Since forces $F_{2}$ and $F_{3}$ depend only on the independent variable $t$, equation (34) can be integrated separately and its solution can be further replaced into equations (31) and (32) (or (33)) that must be solved for the unknowns $u_{N}$ and $u_{G}$. We further make the same assumptions as in subsection 2.1 and use the Hodgkin-Huxley model. Thus, the system of equations of this model is made of equations (31), (32), (34) (without the term involving $\frac{d C}{d u_{N}}$ ), (21) - (24), and the parameters given by formulas (25) - (27).

\section{2-3-Forces Dependent on NO Dynamics}

Lastly, we need to provide expressions for the forces $F_{1}, F_{2}$, and $F_{3}$. These are assumed to depend on the NO dynamics. More precisely, we consider that they have similar shapes to the temporal profiles of the concentrations of $\mathrm{NO}$ in two cases: 1) stepwise activation of $\mathrm{NO}$ without decline in the NO concentration (non-decaying synthesis of $\mathrm{NO}$ ), and 2) dynamic activation of $\mathrm{NO}$ (decaying synthesis of NO). The differential equations corresponding to these two cases are modifications of the Michaelis-Menten equation and were validated experimentally on brain slices in [15].

The equation describing the net production of NO by non-decaying synthesis and inactivation is [15]:

$\frac{d[\mathrm{NO}]}{d t}=v_{1}-\frac{V_{\max }[\mathrm{NO}]}{K_{m}+[\mathrm{NO}]}$,

Where we denoted by $\left[N O\right.$ ] the concentration of $\mathrm{NO}, v_{1}$ the constant rate of $\mathrm{NO}$ synthesis, and $V_{\text {max }}$ the maximum rate at saturating concentration of NO. The constant $K_{m}$ is the concentration of $\mathrm{NO}$ at which the reaction rate is $V_{\max } / 2$.

The equation modelling the dynamic activation of NO and inactivation is [15]:

$\frac{d[\mathrm{NO}]}{d t}=v_{1}\left(1-e^{-k_{1} t}\right) e^{-k_{2} t}-\frac{V_{\max }[\mathrm{NO}]}{K_{m}+[N O]}$,

With $k_{1}$ and $k_{2}$ constant kinetic parameters. The rate of NO activation (synthesis) in equation (36) matches the rate of 
$\mathrm{Ca}^{2+}$-calmodulin binding in dendritic spines after the synaptic stimulation of NMDA receptors [15]. The second term on the right-hand side of equations (35) and (36) represents the rate of NO inactivation.

Analytical solutions to the differential equations (35) and (36) with zero initial condition at $t=0$ were obtained in [25] using He's homotopy perturbation method [26] and are as follows:

$[N O](t)=\frac{v_{1}}{\kappa}\left(1-e^{-\kappa t}\right)$,

Solution to equation (35), and:

$[N O](t)=v_{1}\left(\frac{-e^{-k_{2} t}}{k_{2}-\kappa}+\frac{e^{-\left(k_{1}+k_{2}\right)^{t}}}{k_{1}+k_{2}-\kappa}+e^{-\kappa t}\left(\frac{1}{k_{2}-\kappa}-\frac{1}{k_{1}+k_{2}-\kappa}\right)\right)$,

Solution to equation (36). In formulas (37) and (38) we denoted by $\kappa=\frac{V_{\max }}{K_{m}}$.

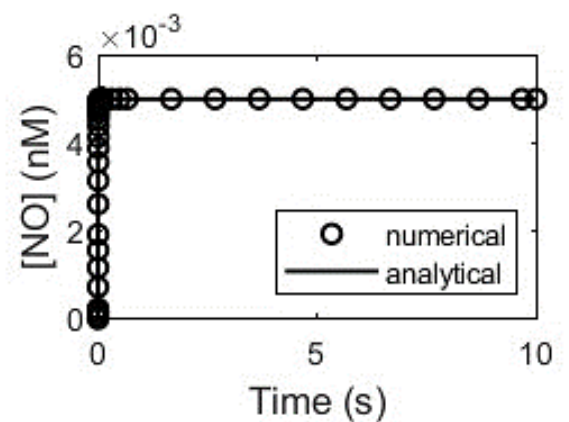

(A)

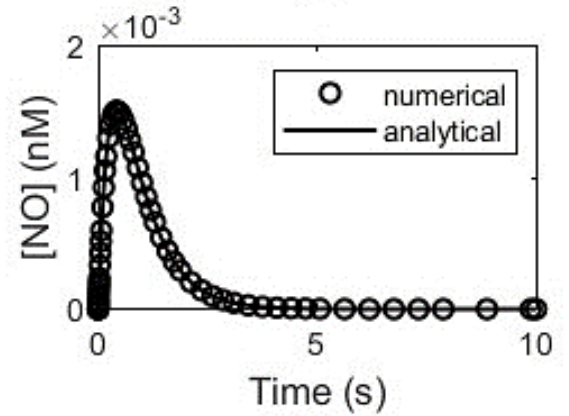

(C)

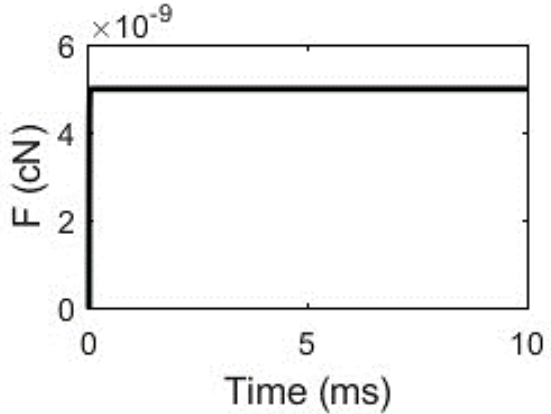

(B)

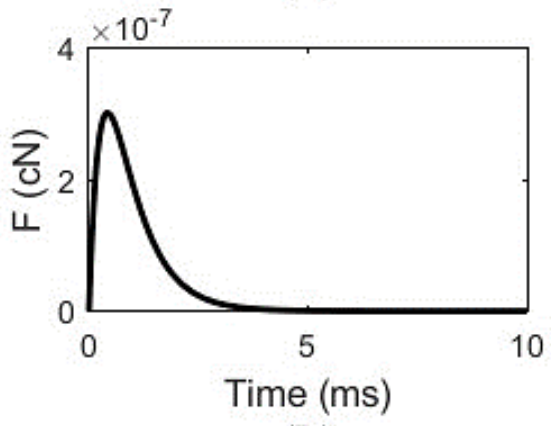

(D)

Figure 5. Profiles of $[N O](\mathrm{A}, \mathrm{C})$ and magnitudes of force $F(\mathrm{~B}, \mathrm{D})$ proportional to [NO]. Formulas (37) (A) and (39) (B) correspond to the case of non-decaying synthesis of and inactivation of NO. Formulas (38) (C) and (40) (D) correspond to the case of dynamic synthesis and inactivation of NO. Numerical solutions to differential equations (35) (A) and (36) (C) are also shown.

In our simulations we used $F_{3}=-F_{2}$ (Figure 4), and $F_{1}=-F_{2}$ (Figure 3 and 4 ). The choice of the directions of these forces is such that vasodilatation happens due to the compression of the spring of constant $k_{G}$, and vasoconstriction is a consequence of vasodilatation and constant length of astrocyte's endfeet. The numerical solution to equation (35) and its corresponding analytical solution (37) are shown in Figure 5(A), while the numerical and analytical solution (38) to equation (36) are plotted in Figure 5(C). These plots were generated using the following [15]:

$v_{1}=1 n M, k_{1}=2 s^{-1}, k_{2}=1.5 s^{-1}, V_{\text {max }}=2000 \frac{n M}{s}, K_{m}=10 n M$.

The numerical solutions were obtained using Matlab's built-in function ode15s. As in [25], we found good agreements between the numerical and analytical solutions for each equation.

We further choose the following expressions for the magnitude of each of the three forces:

$F=0.005 \times 10^{-6}\left(1-e^{-200 t}\right)$,

Corresponding to the case of non-decaying synthesis of NO and shown in Figure 5(B), and:

$F=10^{-6} e^{-1.5 t}\left(1-e^{-2 t}\right)$,

Corresponding to the case of dynamic activation of NO and shown in Figure 5(D). The scaling in formulas (39) and (40) was chosen such that the displacements of the neuron and astrocyte's endfeet found by numerically solving system (19)(24) and the system made of the equations (21)-(24), (31), (32), (34) are physically reasonable. Thus, in our simulations we used $F_{1}=-F_{2}=F_{3}=F$. 


\section{2-4-Parameters}

The parameters used in our simulations are as follows. The thickness of the neuronal membrane is $r=4 \mathrm{~nm}$, the radius of the neuron is $r_{0}=2 \mu \mathrm{m}$ [27], and half of the neuronal mass is $m_{N}=0.1 \mathrm{ng}$ [28]. As in [19-20], we take $k_{0}=$ $0.0013 \mathrm{mg} / \mathrm{ms}^{2}$ and $\eta_{N}=2.5 \times 10^{-5} \mathrm{ng} / \mathrm{ms}$. Also, from formula (27), and the linear relationship between the spring constant $k_{0}$ and Young's modulus $E_{0}$ of the neuron at rest, $k_{0}=\pi r_{0} E_{0}$, we get [19]:

$E=E_{0}\left(1+m^{3}(1-h) n^{4}\right)$,

with $E_{0}=200 \mathrm{~Pa}[24,29]$. From the data published in [30] we estimated the mass of the astrocyte's endfoot to be $m_{G}=1.17 \mathrm{ng}$, and used stiffness measurements from [24] to calculate the spring constant of the endfoot $k_{G}=$ $0.018 \mathrm{mg} / \mathrm{ms}^{2}$. We took $d=10 \mu \mathrm{m}$, approximately the diameter of an astrocyte's soma [31]. The parameters for the modified Hodgkin-Huxley model were adapted from [7]:

$E_{N a}=60 \mathrm{mV}, E_{K}=-88 \mathrm{mV}, E_{C l}=-61 \mathrm{mV}$,

$G_{N a}=0.171 \frac{\mathrm{ms}}{\mathrm{mm}^{2}}, G_{K}=0.1 \frac{\mathrm{ms}}{\mathrm{mm}^{2}}$,

$G_{N a L}=0.000247 \frac{\mathrm{ms}}{\mathrm{mm}^{2}}, G_{K L}=0.0005 \frac{\mathrm{ms}}{\mathrm{mm}^{2}}, G_{C l L}=0.001 \frac{\mathrm{ms}}{\mathrm{mm}^{2}}$.

As in [32], we modelled the effect of NO on the action potential as a $60 \%$ reduction in $G_{K}$ (from a value of $0.25 \frac{\mathrm{mS}}{\mathrm{mm}^{2}}$ ), and a $43 \%$ reduction in $G_{N a}$ (from a value of $0.3 \frac{\mathrm{ms}}{\mathrm{mm}^{2}}$ ). In all numerical simulations we applied a constant external current per unit surface area $I=0.1 \mu \mathrm{A} / \mathrm{mm}^{2}$. Lastly, using the value of the specific membrane capacitance of $0.01 \mu \mathrm{F} / \mathrm{mm}^{2}$ at mechanical equilibrium $\left(u_{N}=0\right)$ and formula (26), we get:

$c_{m}=0.01\left(1-\frac{u_{N}}{r}\right) \frac{\mu F}{m m^{2}}$.

System (19) - (24) and the system made of the equations (21) - (24), (31), (32), (34) were both solved using the following initial conditions:

$$
\begin{aligned}
& V(0)=-65 m V, m(0)=\frac{\alpha_{m}(V(0))}{\alpha_{m}(V(0))+\beta_{m}(V(0))}, \\
& n(0)=\frac{\alpha_{n}(V(0))}{\alpha_{n}(V(0))+\beta_{n}(V(0))}, h(0)=\frac{\alpha_{h}(V(0))}{\alpha_{h}(V(0))+\beta_{h}(V(0))}, \\
& u_{N}(0)=0 \mu m, \dot{u}_{N}(0)=0 \frac{\mu m}{m s}, u_{G}(0)=0 \mu m, \dot{u}_{G}(0)=0 \frac{\mu m}{m s} .
\end{aligned}
$$

\begin{tabular}{|c|c|c|}
\hline Parameters & Values and Units & Descriptions \\
\hline$r$ & $4 \mathrm{~nm}$ & Thickness of neuronal membrane \\
\hline$r_{0}$ & $2 \mu m$ & Radius of a neuron \\
\hline$m_{N}$ & $0.1 n g$ & Half of a neuron's mass \\
\hline $\boldsymbol{k}_{\mathbf{0}}$ & $0.0013 \mathrm{mg} / \mathrm{ms}^{2}$ & Spring constant of a neuron at rest \\
\hline $\boldsymbol{E}_{\mathbf{0}}$ & $200 P a$ & Young's modulus of a neuron at rest \\
\hline$\eta_{N}$ & $2.5 \times 10^{-5} \mathrm{ng} / \mathrm{ms}$ & Damping coefficient of a neuron \\
\hline$d$ & $10 \mu m$ & Sum of the lengths of an astrocyte's endfeet at mechanical equilibrium \\
\hline $\boldsymbol{k}_{G}$ & $0.018 \mathrm{mg} / \mathrm{ms}^{2}$ & Spring constant of an astrocyte's endfoot \\
\hline$m_{G}$ & $1.17 n g$ & Mass of an astrocyte's endfoot \\
\hline$E_{N a}$ & $60 m V$ & Reversal potential of $\mathrm{Na}^{+}$ \\
\hline $\boldsymbol{E}_{K}$ & $-88 m V$ & Reversal potential of $K^{+}$ \\
\hline $\boldsymbol{E}_{C l}$ & $-61 m V$ & Reversal potential of $\mathrm{Cl}^{-}$ \\
\hline$G_{N a}$ & $0.171 \mathrm{mS} / \mathrm{mm}^{2}$ & Maximal conductance of $\mathrm{Na}^{+}$Current (NO effect) \\
\hline$G_{K}$ & $0.1 \mathrm{mS} / \mathrm{mm}^{2}$ & Maximal conductance of $K^{+}$Current (NO effect) \\
\hline$G_{N a L}$ & $0.000247 \mathrm{mS} / \mathrm{mm}^{2}$ & Conductance of leak $\mathrm{Na}^{+}$current \\
\hline$G_{K L}$ & $0.0005 \mathrm{mS} / \mathrm{mm}^{2}$ & Conductance of leak $K^{+}$current \\
\hline$G_{C l L}$ & $0.001 \mathrm{mS} / \mathrm{mm}^{2}$ & Conductance of leak $\mathrm{Cl}^{-}$current \\
\hline$c_{m}$ & $0.01 \mu F / \mathrm{mm}^{2}$ & Specific capacitance of neuronal membrane at mechanical equilibrium \\
\hline
\end{tabular}

Table 1. List of parameters with corresponding descriptions, values and units.

In addition, for the model involving the astrocyte with two endfeet, equation (34) reduces to $\ddot{x}=0$ since $F_{3}=-F_{2}$, 
which can be easily integrated to get: $x(t)=\dot{x}(0) t+x(0)$. Noticing that zero initial displacement and speed for $x(t)$ will make $x(t)=0, \forall t \geq 0$, and thus reduce the model of astrocyte with two endfeet to the one involving an astrocyte with only one endfoot, in our simulations we took:

$x(t)=(0.01 t+10) \mu m$.

We solved system (19) - (24) and the system made of the equations (21) - (24), (31), (32), (34) numerically using Matlab's built-in function ode15s with its default values for the relative error tolerance $\left(10^{-3}\right)$ and absolute error tolerance $\left(10^{-6}\right)$. The algorithm implemented in this solver involves a modified linear multistep backward difference formula of order up to 5 that has good stability, and an adaptive step size that changes according to a numerical scheme that calculates relative and absolute error tolerances [33].

A summary of the parameters used in the models is given in Table 1. Table 2 summarizes the initial conditions used in our numerical simulations.

Table 2. List of initial conditions for the dependent variables of the models and their descriptions.

\begin{tabular}{lll}
\hline Dependent Variables & Initial Values and Units & Descriptions \\
\hline $\boldsymbol{u}_{N}$ & $0 \mu m$ (formulas (44)) & neuronal displacement \\
$\dot{\boldsymbol{u}}_{N}$ & $0 \mu \mathrm{m} / \mathrm{ms}$ (formulas (44)) & neuronal speed \\
$\boldsymbol{u}_{G}$ & $0 \mu \mathrm{m}$ (formulas (44)) & displacement of astrocyte's endfoot \\
$\dot{\boldsymbol{u}}_{\boldsymbol{G}}$ & $0 \mu \mathrm{m} / \mathrm{ms}$ (formulas (44)) & speed of astrocyte's endfoot \\
$\boldsymbol{V}$ & $-65 \mathrm{mV}$ (formulas (44)) & electric potential \\
$\boldsymbol{m}$ & $3.76951 \cdot 10^{-13}$ (formulas (25) and (44)) & displacement of activated $\mathrm{Na}^{+}$channel \\
$\boldsymbol{n}$ & 0.05182 (formulas (25) and (44)) & displacement of activated $\mathrm{K}^{+}$channel \\
$\boldsymbol{h}$ & 0.99325 (formulas (25) and (44)) & displacement of inactivated $\mathrm{Na}^{+}$channel \\
\hline
\end{tabular}

\section{3- Results}

The profiles of the voltage $V$, displacements $m, n, h$, and Young's modulus $E$ (formula (41)) for the two models (astrocyte with one endfoot and astrocyte with two endfeet) and the two forces given by formulas (39) and (40) are shown in Figure 6. The NO-controlled mechanics of both models does not change the classical electrical behaviour of the neuronal membrane.

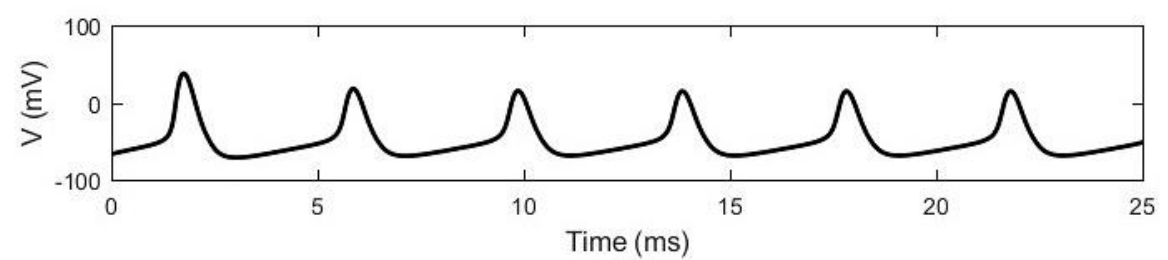

(A)

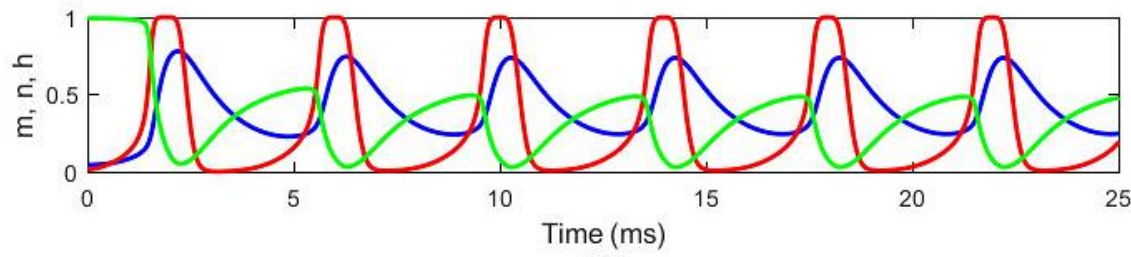

(B)

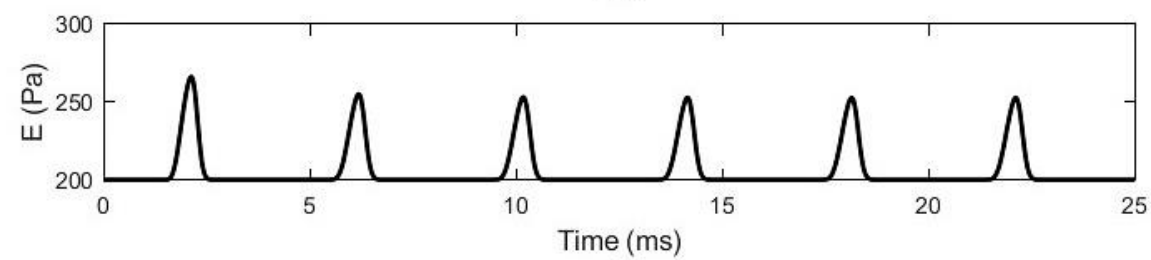

(C)

Figure 6. Results for both models of brain-on-a-chip and for both cases of NO synthesis (non-decaying and dynamic): (A) voltage $V$, (B) displacements $n$ (blue line), $m$ (red line), and $h$ (green line), (C) Young's modulus $E$.

Figures 7 and 8 show the profiles of the displacements $u_{N}$ and $u_{G}$ in the brain-on-a-chip with an astrocyte having one endfoot. Figure 7 corresponds to the force profile (39), and Figure 8 shows the results for the force profile (40). While the oscillatory behaviour of the neuronal displacement is similar for both force profiles (Figures 7(A) and 8(A), 
and their corresponding zoom-ins showed in Figures 7(B) and 8(B)), the displacement of the astrocyte's endfoot has different profiles for the two force shapes (Figures 7(C, D) and $8(\mathrm{C}, \mathrm{D})$ ). If the applied external forces of opposite directions acting on the endfoot have the magnitude given by formula (39), then $u_{G}$ is negative and reaches a steady state of approximately $-2.5 \mu \mathrm{m}$ (Figure 7(C)). The oscillations in $u_{G}$ are very small in this case (Figure 7(D)). On the other hand, if the opposite external forces have the magnitude given by formula (40), then a sharp, but small, decrease in $u_{G}$ is observed, after which $u_{G}$ oscillates rapidly around the zero steady state (Figures $8(\mathrm{C}, \mathrm{D})$ ).

The profiles of the displacements $u_{N}$ and $u_{G}$ in the brain-on-a-chip with an astrocyte having two endfeet are presented in Figure 9 (for the force profile (39)), and Figure 10 (for the force profile (40)). We notice again the rapid oscillations of $u_{N}$ for both force shapes (Figures 9 (A, B) and $10(\mathrm{~A}, \mathrm{~B})$ ). The displacement $u_{G}$ slightly oscillates towards a negative steady state at $-2.5 \mu \mathrm{m}$ (Figure $9(\mathrm{C})$ ) for the force profile (39), while for the profile (40), the displacement rapidly oscillates, and an initial fast decrease in $u_{G}$ is followed by a linear increase (Figure $10(\mathrm{C})$ ).

\section{4- Discussion}

Our results suggest the following possible mechanisms for the proposed brain-on-a-chip. If we identify the externally applied forces with the action of $\mathrm{NO}$ on the neuro-glial-vascular unit and the deformation of astrocyte's endfeet with the movement of the vascular wall, then the dynamic activation of $\mathrm{NO}$ and inactivation produces a sudden vasodilatation (negative displacement $u_{G}$ ) during the NO synthesis followed by either small oscillations around the undeformed steady state (model with an astrocyte having one endfoot) or vasoconstriction of both endfeet (positive displacements $u_{G}$ and $x$ predicted by the model with an astrocyte having two endfeet). In the case of non-decaying synthesis and inactivation of NO, both models predict a permanent vasodilatation which might be a contributing factor in the onset of a microaneurysm. We notice that the behaviour of $u_{G}$ did not correlate to the deformation of the neuron and that the changes in maximal conductances due to NO action and the steady and small oscillations of $u_{N}$ did not inhibit the action potentials.

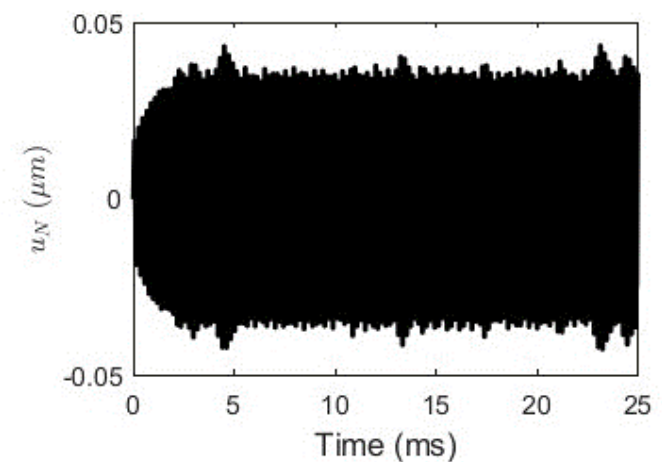

(A)

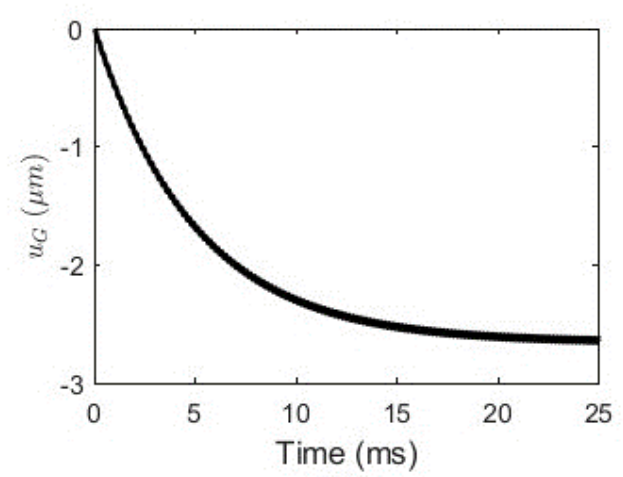

(C)

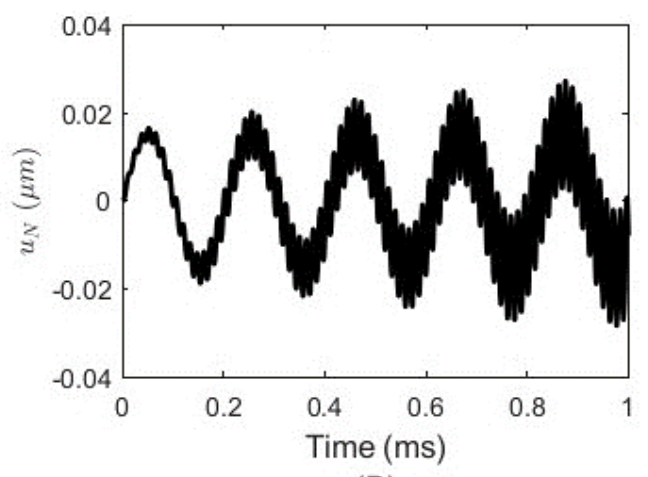

(B)

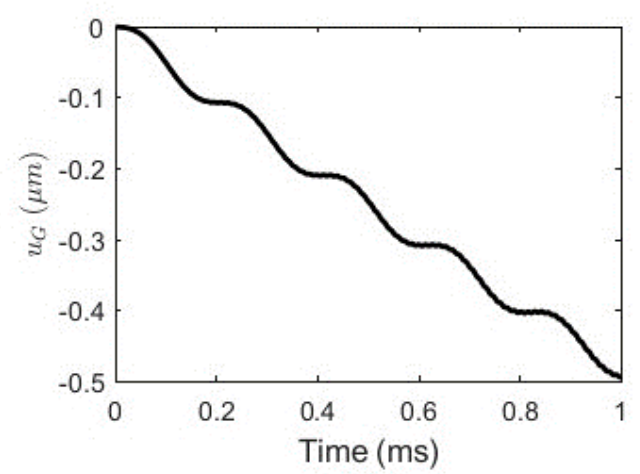

(D)

Figure 7. Results for the brain-on-a-chip with one glia's endfoot in the case of non-decaying synthesis of NO: (A) neuronal displacement $u_{N}$, (B) a zoom-in of $u_{N}$, (C) displacement of the endfoot $u_{G}$, (D) a zoom-in of $u_{G}$. 


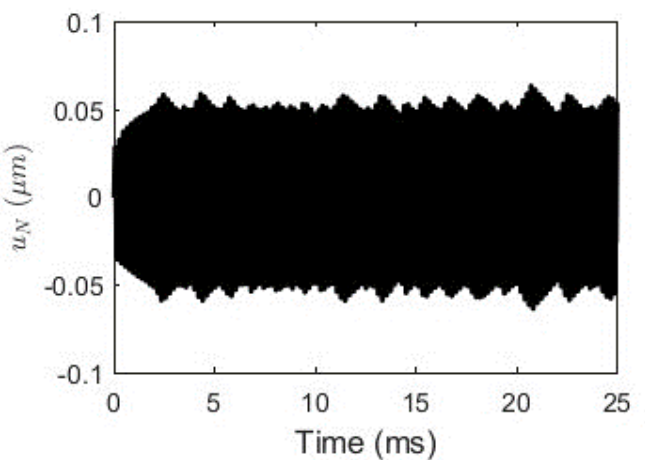

(A)

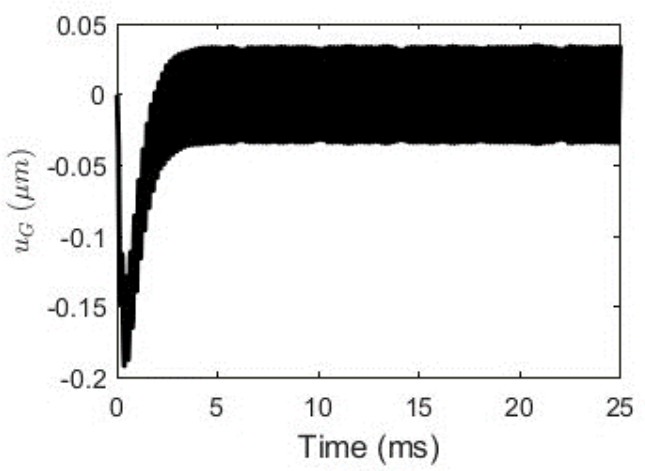

(C)

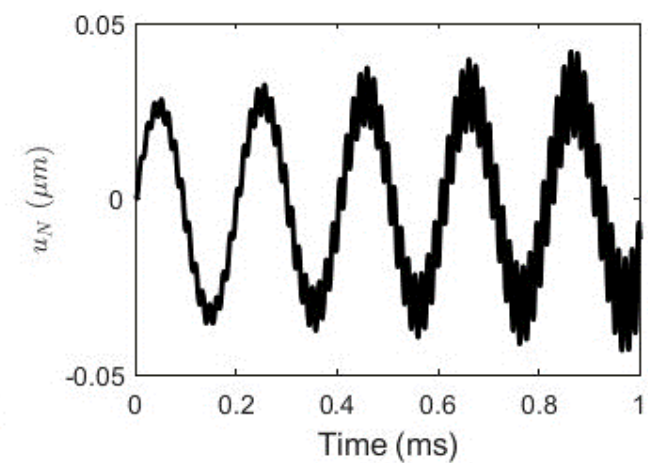

(B)

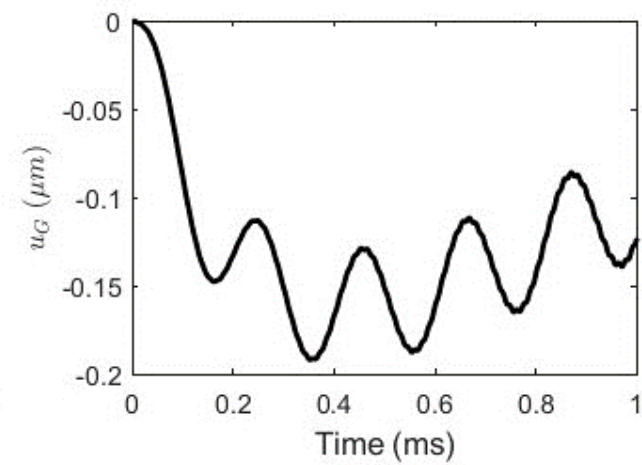

(D)

Figure 8. Results for the brain-on-a-chip with one glia's endfoot in the case of dynamic synthesis of NO: (A) neuronal displacement $u_{N}$, (B) a zoom-in of $u_{N}$, (C) displacement of the endfoot $u_{G}$, (D) a zoom-in of $u_{G}$.

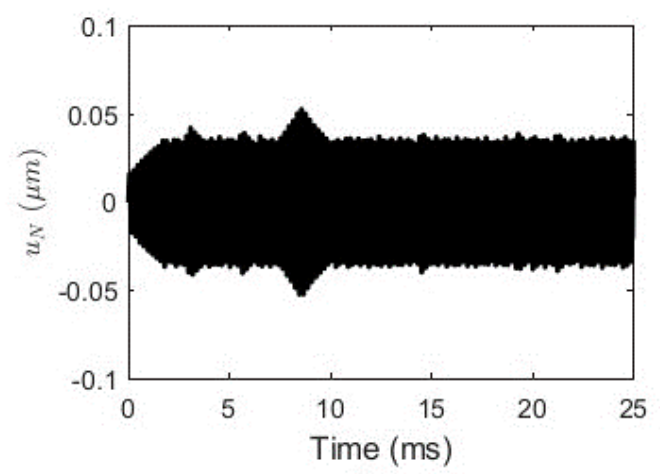

(A)

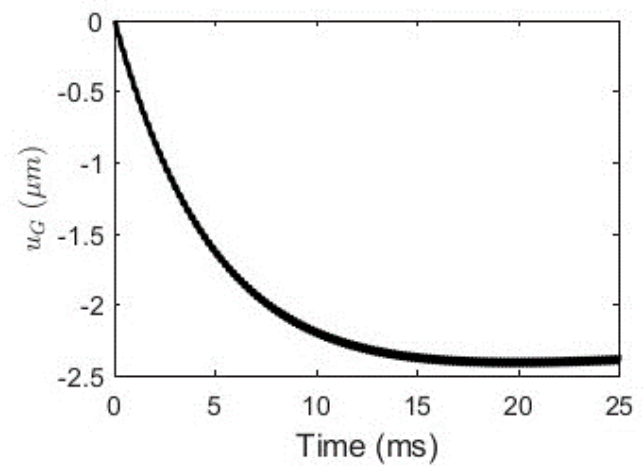

(C)

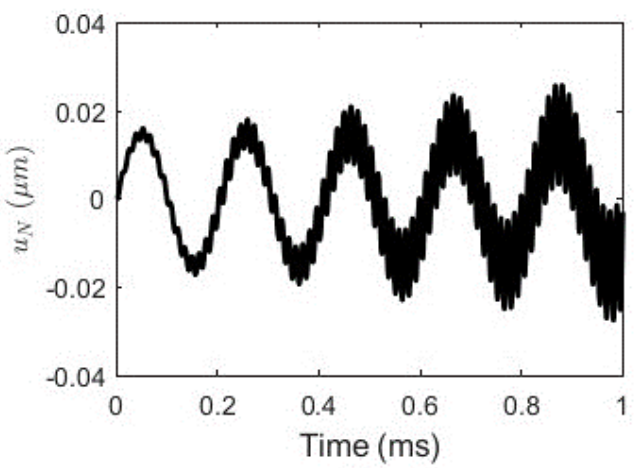

(B)

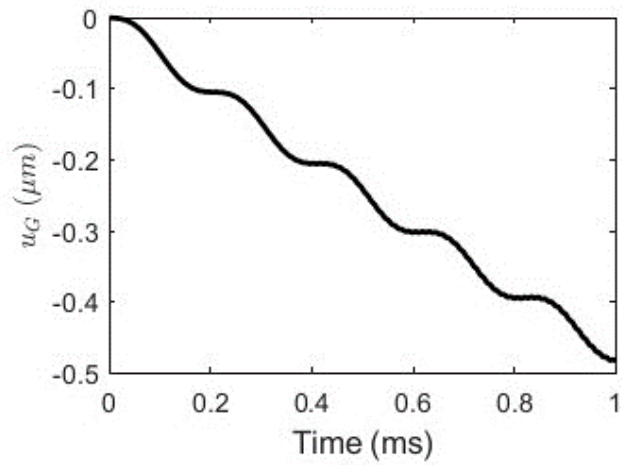

(D)

Figure 9. Results for the brain-on-a-chip with two glia's endfeet in the case of non-decaying synthesis of NO: (A) neuronal displacement $u_{N}$, (B) a zoom-in of $u_{N}$, (C) displacement of the endfoot $u_{G}$, (D) a zoom-in of $u_{G}$. 


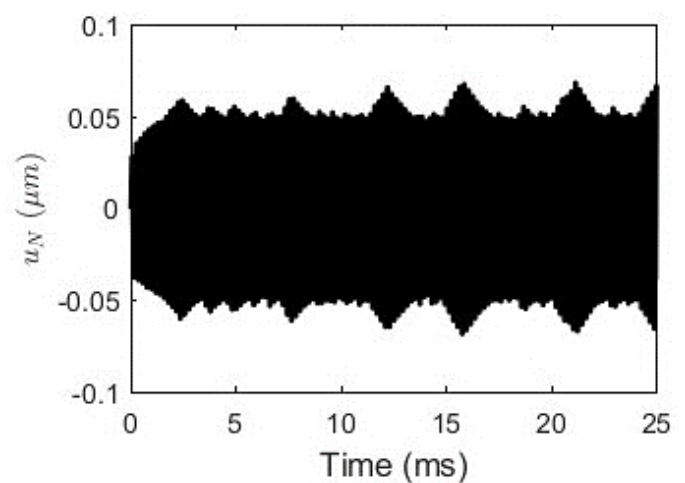

(A)

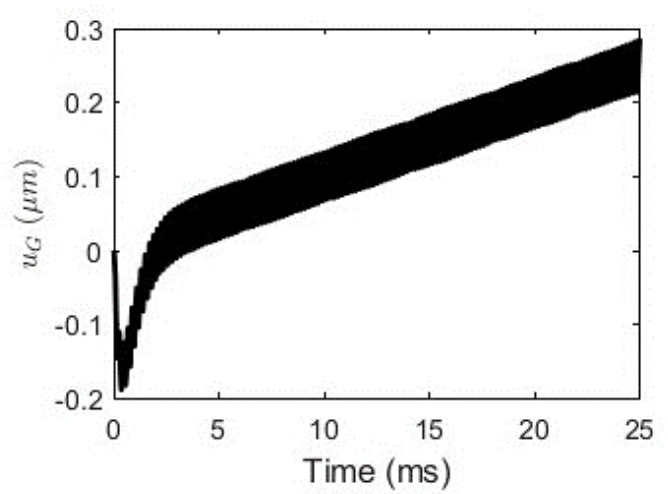

(C)

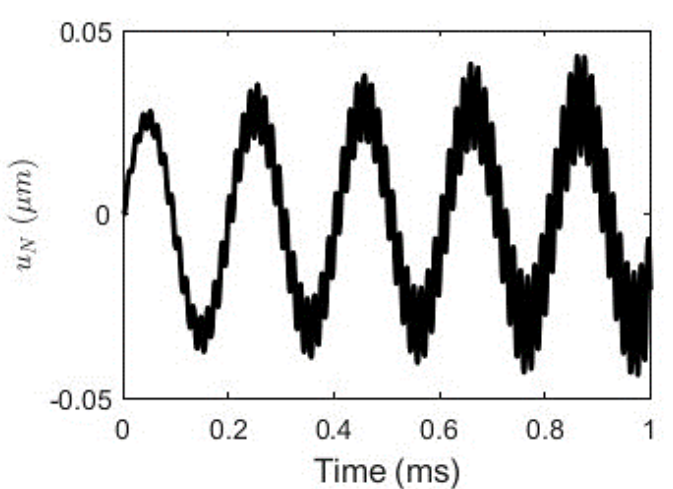

(B)

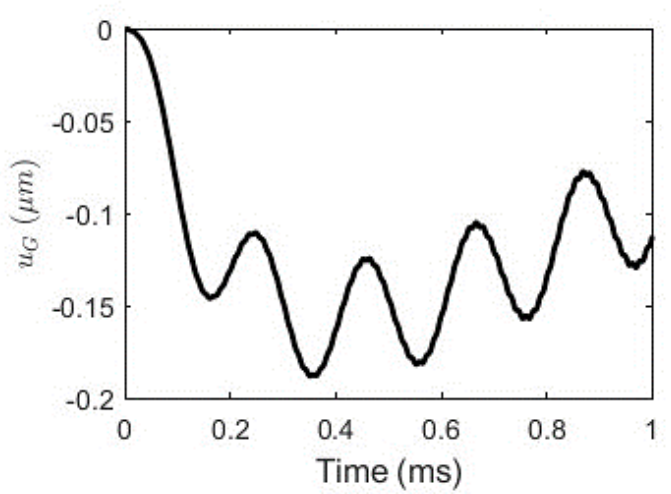

(D)

Figure 10. Results for the brain-on-a-chip with two glia's endfeet in the case of dynamic synthesis of NO: (A) neuronal displacement $u_{N}$, (B) a zoom-in of $u_{N}$, (C) displacement of the endfoot $u_{G}$, (D) a zoom-in of $u_{G}$.

There are currently no published experimental observations or other numerical simulations that we could use to validate and compare our results to. However, there are some indirect inferences that can be made from existing literature. For instance, the studies in [11-12, 15, 17, 34] report measurements of time dynamics and diffusion of NO in brain tissue. A review of the corresponding mathematical models describing the spatio-temporal NO biotransport in brain can be found in [35]. In this paper we used the mathematical models of decaying and non-decaying NO syntheses proposed by Hall and Garthwaite [15] and a trial and error approach to represent external forces dependent on the NO dynamics. Hall and Garthwaite validated their models using indirect measurements of NO levels in intact brain tissues from rats. Their immunohistochemical analysis showed that the mechanism of NO-degrading in brain is different from some established breakdown pathways such as reaction with haemoglobin in red blood cells, superoxide, molecular oxygen and lipid peroxyl radicals. While Hall and Garthwaite were not able to characterize this newly found mechanism, the experiments performed more recently in rat brain in vivo using NO-selective carbon fiber microelectrodes suggested that most of the NO diffusion in brain is intracellular rather than extracellular [34].

While the above-mentioned studies focused entirely on the role played by neuronal NO in neurotransmission, Metea and Newman [36] and the review by Petzold and Murthy [6] present experimental observations of the NO involvement in the vasomotor regulation of cerebral blood vessels. The experiments by Metea and Newman were performed on isolated whole-mount retina of rats. The retina was exposed to either light stimulation or chemically-driven glial stimulation. During the experiments, the NO concentration was controlled and monitored using NO-sensitive electrodes. Enhanced retinal images were used to manually measure the diameters of arterioles. The results showed that regardless of the type of stimulation, the vessels either dilated or contracted by $10 \%-50 \%$. It was observed that NO controlled light-evoked vasomotor responses. The durations of vasodilatation and vasoconstriction were dependent on the chemical manipulations performed during experiments and the NO concentration. During light stimulation the arterioles either dilated by approximately $2 \mu \mathrm{m}$ or contracted by $1-1.5 \mu \mathrm{m}$. In our models we identified the movements of the astrocyte's endfeet with the vasomotor responses of an arteriole. Figures $7(\mathrm{C})$ and $9(\mathrm{C})$ show vasodilatations of about $2.5 \mu \mathrm{m}$ in the case of non-decaying synthesis and inactivation of NO, which are close to those observed during light stimulation of the retina. In the case of dynamic synthesis and inactivation of $\mathrm{NO}$, the vasodilatations and vasocontractions predicted by our models were approximately $0.2 \mu \mathrm{m}$ (Figures 8(C) and 10(C)) which are less than those reported by Metea and Newman [36]. Since our models do not account for light effects which enhance the vasomotor responses of retinal arterioles, we believe that our predictions are reasonable. No mathematical models capable to predict the vasoactive behaviour observed experimentally have been published yet. The rapid oscillations seen in Figures 8(C) and 10(C) can only be explained as artefacts caused by the combination between the high-frequency 
oscillations of the neuronal displacement (Figures 7-10(A)) and the fast, transient variation of the applied force given by formula (40) (Figure 5(D)). Although $\mathrm{Ca}^{2+}$ signals measured in neurons and astrocytes are characterized by rapid oscillations and can be directly related to vessel diameter in ex vivo preparations, no correlation between $\mathrm{Ca}^{2+}$ dynamics and vasomotor responses has been found experimentally yet $[6,36]$, and thus the existence of fast vasoactive oscillations cannot be justified in this manner. In addition, in our models the $\mathrm{Ca}^{2+}$ dynamics is not even considered. Lastly, we notice that there are no published experimental and/or theoretical studies of the possible role played by the neuronal NO in the onset of cerebral microaneurysms. Thus, the prediction made by our models suggesting that a non-decaying synthesis and inactivation of NO may contribute to the formation of a microaneurysm (Figures $7(C)$ and $9(\mathrm{C})$ ) needs experimental validation.

The high amplitude oscillations of the neuronal displacement seen in Figures 7-10(A) do not appear to have the sharp mechanical spikes that correlate to action potentials and were observed experimentally by Kim et al [37] and predicted by a mathematical model of El Hady and Machta [38] (Other phenomenological models of neuronal volumetric changes during action potentials are based solely on transport of chemical species across the membrane and do not account for neuronal mechanics (see [7] and references within for such models). Thus, we do not consider these models in our discussion). Kim et al [37] used a high bandwidth atomic force microscope to measure the nanometer-scale movements of the nerve terminals of the mouse neurohypophysis caused by action potentials. A rapid, transient displacement of $5-10 \mathrm{~nm}$ that is in phase and has almost the same duration with the action potential is measured. If the nerve has about 100 terminals, then the radius of one terminal (neuron) increases by only $0.025 \mathrm{~nm}$ during an action potential. The mathematical model in [38] uses a fluid mechanics approach to predict displacements of the same order of magnitude as seen experimentally. On the other hand, the neuronal displacements predicted by our models are about $50 \mathrm{~nm}$, about 2,000 times bigger than the experimentally observed mechanical spikes during action potentials. One reason for this discrepancy could be the proposed expression for the dynamic spring constant of the viscoelastic element modelling the neuron. Formula (27) was obtained by a trial and error process so that the spring constant matched the spring constant of a neuron estimated from measurements. In addition, formula (27) has not been validated experimentally yet. However, finding a more accurate expression could be difficult due to the utilization of the modified Hodgkin-Huxley model. The very finely adjusted equations and parameters of the Hodgkin-Huxley model might hide relevant information about the mechanics of the neuronal membrane. This makes the coupling of the model with the neuronal mechanics difficult to accomplish. The use of the mechano-electric analogy could allow us to reinterpret the Hodgkin-Huxley model using mechanical descriptors which are easier to combine with other mechanical and hydraulic elements. Most likely a chemically-driven, mechano-hydraulic system could provide improved, more accurate predictions.

\section{5- Conclusions and Future Work}

In this paper we proposed a brain-on-a-chip for studying the effects of NO on vasodilatation via glia's endfeet. The components of this chip were a neuron and it membrane, and the endfeet of an astrocyte assumed to be connected to the vascular wall of an arteriole. The neuron was represented by a linear viscoelastic Kelvin-Voigt solid, while the ionic gates of the neuronal membrane were modelled as linear viscoelastic Maxwell fluids. The astrocyte was modelled as either one endfoot or two endfeet. The endfoot responsible for vasodilatation was modelled as a spring-mass system, while the second endfoot controlling vasoconstriction was assumed to be the second branch of an analog of an Atwood machine with a pulley-like astrocyte's soma. The action of NO on the proposed brain-on-a-chip was modelled as forces proportional to the concentration of NO predicted by models of NO synthesis and inactivation which were validated experimentally on brain slices. Since the ionic transport across neuronal membrane was modelled using the HodgkinHuxley equations, the effects of NO on the action potentials was represented as a reduction in the maximum conductances of $\mathrm{Na}^{+}$and $\mathrm{K}^{+}$. In our numerical simulations we considered two cases: 1) non-decaying synthesis of NO and inactivation, and 2) dynamic synthesis of $\mathrm{NO}$ and inactivation. Our results show an oscillatory displacement of the neuron in both cases. However, the endfoot controlling vasodilatation experiences different behaviours: in the case of non-decaying $\mathrm{NO}$ synthesis the spring contracts to a new deformed steady state corresponding to a possibly permanent vasodilatation, and in the case of dynamic activation of NO the vasodilatation happens fast, the shape of the endfoot's displacement mimicking that of the NO dynamics. The permanent vasodilatation predicted in the case of non-decaying NO activation could lead to the formation of a microaneurysm. Lastly, the only difference between the chip with one endfoot and the one with two endfeet was the vasoconstriction done by both endfeet in the case of dynamic activation of NO. The fact that both endfeet compress the vascular wall after the cessation of NO synthesis might be a consequence of the fact that the vasoconstrictive endfoot was modelled as a pure mechanical reaction to the deformation of the springmass system driving the vasodilatation, no other mechano-chemical controllers of vasoconstriction were accounted for in the model.

In our future work we plan to incorporate in our models of brain-on-a-chip the dynamics of $\mathrm{Ca}^{2+}$ and account for the role of NO in the regulation of blood flow and oxygen delivery. Lastly, the addition of spatial variability will provide a clearer view on the possible role played by a non-decaying activation of $\mathrm{NO}$ in the formation and growth of microaneurysms in the brain. 


\section{6- Conflict of Interest}

The author declares no conflict of interest.

\section{7- References}

[1] Attwell, D., A. Buchan, S. Charpak, M. Lauritzen, B.A. MacVicar, and E.A. Newman. "Glial and Neuronal Control of Brain Blood Flow." Nature 468, (2010): 232-243. doi: 10.1038/nature09613.

[2] Contestabile, A., B. Monti, and E. Polazzi. "Neuronal-Glial Interactions Define the Role of Nitric Oxide in Neural Functional Processes.” Curr. Neuropharmacol. 10, no. 4 (2012): 303-310. doi: 10.2174/157015912804143522.

[3] Gordon, G.R.J., S.J. Mulligan, and B.A. MacVicar. “Astrocyte Control of the Cerebrovasculature.” Glia 55 (2007): 1214-1221. doi: 10.1002/glia.20543.

[4] Gordon, G.R., C. Howarth, and B.A. MacVicar. "Bidirectional Control of Arteriole Diameter by Astrocytes." Exp. Physiol. 96, no. 4 (2011): 393-399. doi: 10.1113/expphysiol.2010.053132.

[5] Mishra, A. (2017). "Binaural Blood Flow Control by Astrocytes: Listening to Synapses and the Vasculature.” J. Physiol. 595, no. 6 (2017): 1885-1902. doi: 10.1113/JP270979.

[6] Petzold, G.C. and V.N. Murthy. "Role of Astrocytes in Neurovascular Coupling." Neuron 71, no. 5 (2011): 782-797. doi: 10.1016/j.neuron.2011.08.009.

[7] Wei, Y., G. Ullah, and S.J. Schiff, S.J. "Unification of Neuronal Spikes, Seizures, and Spreading Depression." The Journal of Neuroscience 34, no. 35 (2014):11733-11743. doi: 10.1523/JNEUROSCI.0516-14.2014.

[8] Drapaca, C.S. "Brain-on-a-Chip: Design and Modeling.” DCDIS Series B: Applications \& Algorithms 25, no. 3-4 (2018): 147162.

[9] Aebersold, M.J., H. Dermutz, C. Forró, S. Weydert, G. Thompson-Steckel, J. Vörös, and L. Demkó. "Brains on a chip: Towards Engineered Neural Networks.” TrAC Trends in Analytical Chemistry 78, (2016): 60-69. doi: 10.1016/j.trac.2016.01.025.

[10] Soscia, D., A. Belle, N. Fischer, H. Enright, A. Sales, J. Osburn, W. Benett, E. Mukerjee, K. Kulp, S. Pannu, and E. Wheeler. “Controlled Placement of Multiple CNS Cell Populations to Create Complex Neuronal Cultures.” PLoS ONE 12, no. 11 (2017): e0188146. https://doi.org/10.1371/journal.pone.0188146.

[11] Barbosa, R.M., C.F. Lourenco, R.M. Santos, F. Pomerleau, P. Huettl, G.A. Gehardt, and J. Laranjinha. "In Vivo Real-Time Measurement of Nitric Oxide in Anesthetized Rat Brain.” Methods in Enzymology 441, (2008): 351-367. doi: 10.1016/S00766879(08)01220-2.

[12] Buerk, D.G., B.M. Ances, J.H. Greenberg, and J.A. Detre. "Temporal Dynamics of Brain Tissue Nitric Oxide during Functional Forepaw Stimulation in Rats." NeuroImage 18, (2003): 1-9.

[13] Moncada, S., R.M.J. Palmer, and E.A. Higgs. (1991). "Nitric Oxide: Physiology, Pathophysiology, and Pharmacology." Pharmacological Reviews 43, no. 2 (1991): 109-142.

[14] Garry, P.S., M. Ezra, M.J. Rowland, J. Westbrook, and K.T.S. Pattinson. "The Role of the Nitric Oxide Pathway in Brain Injury and Its Treatment - From Bench to Bedside." Exp. Neurol. 263, (2015): 235-243. doi: 10.1016/j.expneurol.2014.10.017.

[15] Hall, C.N. and J. Garthwaite. (2006). “Inactivation of Nitric Oxide by Rat Cerebellar Slices.” J. Physiol. 577, no. 2 (2006): 549567. doi: 10.1113/jphysiol.2006.118380.

[16] Helms, C.C., X. Liu, and D.B. Kim-Shapiro. "Recent Insights into Nitrite Signaling Processes in Blood.” Biol. Chem. 3, (2016): 319-329. doi: 10.1515/hsz-2016-0263.

[17] Ledo, A., R.M. Barbosa, G.A. Gerhardt, E. Cadenas, and J. Laranjinha. "Concentration Dynamics of Nitric Oxide in Rat Hippocampal Subregions Evoked by Stimulation of the NMDA Glutamate Receptor.” Proc. Natl. Acad. Sci. USA 102, no. 48 (2005): 17483-17488. doi: 10.1073/pnas.0503624102.

[18] Santos, R.M., C.F. Lourenco, A. Ledo, R.M. Barbosa, and J. Laranjinha. "Nitric Oxide Inactivation Mechanisms in the Brain: Role in Bioenergetics and Neurodegeneration.” Int J Cell Biol. 2012, (2012): 391914. doi: 10.1155/2012/391914.

[19] Drapaca, C.S. "An Electromechanical Model of Neuronal Dynamics using Hamilton's Principle.” Frontiers in Cellular Neuroscience 9, (2015): 271. doi: 10.3389/fncel.2015.00271.

[20] Drapaca, C.S. "Fractional Calculus in Neuronal Electromechanics.” J. Mech. Materials Struct. 12, no. 1 (2017): 35-55. doi: 10.2140/jomms.2017.12.35.

[21] Hodgkin, A.L. and A.F. Huxley. "A Quantitative Description of Membrane Current and its Application to Conduction and Excitation in Nerve.” J. Physiol. (Lond.) 117, no. 4 (1952): 500-544.

[22] Sykova, E., and C. Nicholson. "Diffusion in Brain Extracellular Space." Physiol. Rev. 88, (2008): 1277-1340. doi: 10.1152/physrev.00027.2007.

[23] Hille, Bertil. "Ion Channels of Excitable Membranes. Third Edition” Sinauer Associates, Sunderland, MA (July 2001).

[24] Zou, S., R. Chisholm, J.S. Tauskela, G.A. Mealing, L.J. Johnston, and C.E. Morris. "Force Spectroscopy Measurements Show that Cortical Neurons Exposed to Excitotoxic Agonists Stiffen before Showing Evidence of Bleb Damage.” PLOS ONE 8, no. 
8 (2013): e73499. doi: 10.1371/journal.pone.0073499.

[25] Mehala, N. and L. Rajendran. "Analytical Solutions of Nonlinear Differential Equations in the Mathematical Model for Inactivation of Nitric Oxide by Rat Cerebellar Slices." American Journal of Analytical Chemistry 5, (2014): 908-919. doi: 10.4236/ajac.2014.514099.

[26] He, J.-H. "A Coupled Method of a Homotopy Technique and a Perturbation Technique for Non-Linear Problems." International Journal of Non-Linear Mechanics 35, no. 1 (2000): 37-43. doi: 10.1016/S0020-7462(98)00085-7.

[27] Dayan, Peter and Laurence F. Abbott. "Theoretical Neuroscience Computational and Mathematical Modeling of Neural Systems.” The MIT Press (September 1, 2005).

[28] Corbin, E. A., L.J. Millet, K.R. Keller, W.P. King, and R. Bashir. "Measuring Physical Properties of Neuronal and Glial Cells with Resonant Microsensors.” Anal. Chem. 86, (2014): 4864-4872.

[29] Lu, Y.B., K. Franze, G. Seifert, C. Steinhauser, F. Kirchhoff, H. Wolburg, J. Guck, P. Janmey, E.Q. Wei, J. Kas, and A. Reichenbach. "Viscoelastic Properties of Individual Glial Cells and Neurons in the CNS." Proc. Natl. Acad. Sci. U.S.A. 103, no. 47 (2006): 17759-17764. doi: 10.1073/pnas.0606150103.

[30] Mota, B. and S. Herculano-Houzel. "All Brains are Made of This: A Fundamental Building Block of Brain Matter with Matching Neuronal and Glial Masses.” Frontiers in Neuroanatomy 8 (2014): 127. doi: 10.3389/fnana.2014.00127.

[31] Ventura, R.E. (2018). Astrocytes, https://synapseweb.clm.utexas.edu/astrocytes.

[32] Steinert, J.R., C. Kopp-Scheinpflug, C. Baker, R.A.J. Challiss, R. Mistry, M.D. Haustein, S.J. Griffin, H. Tong, B.P. Graham, and I.D. Forsythe. "Nitric Oxide is a Volume Transmitter Regulating Postsynaptic Excitability at a Glutamatergic Synapse." Neuron 60, (2008): 642-656. doi: 10.1016/j.neuron.2008.08.025.

[33] Shampine, L.F. and P. Bogacki. “The Effect of Changing the Stepsize in Linear Multistep Codes.” SIAM J. Sci. Statist. Comput. 10, no. 5 (1989): 1010-1023.

[34] Laranjinha, J., R.M. Santos, C.F. Lourenco, A. Ledo, and R.M. Barbosa. "Nitric Oxide in the Brain: Translation of Dynamics into Respiration Control and Neurovascular Coupling." Ann. N.Y. Acad. Sci. 1259, (2012): 10-18. doi: 10.1111/j.17496632.2012.06582.x.

[35] Buerk, D.G. "Can We Model Nitric Oxide Biotransport? A Survey of Mathematical Models for a Simple Diatomic Molecule with Surprisingly Complex Biological Activities.” Annu. Rev. Biomed. Eng. 3, (2001): 109-143.

[36] Metea, M.R. and E.A. Newman. "Glial Cells Dilate and Constrict Blood Vessels: A Mechanism of Neurovascular Coupling." The Journal of Neuroscience 26, no. 11 (2006): 2862-2870. doi: 10.1523/JNEUROSCI.4048-05.2006.

[37] Kim, G.H., P. Kosterin, A.L. Obaid, and B.M. Salzberg. “A Mechanical Spike Accompanies the Action Potential in Mammalian Nerve Terminals.” Biophysical Journal 92, (2007): 3122-3129. doi: 10.1529/biophysj.106.103754.

[38] El Hady, A. and B.B. Machta. "Mechanical Surface Waves Accompany Action Potential Propagation." Nature Communications 6 (2015): 6697. doi: 10.1038/ncomms7697. 